\title{
Use of Quantitative Pharmacology in the Development of HAE1, a High-Affinity Anti-IgE Monoclonal Antibody
}

\author{
Wendy S. Putnam, ${ }^{1,5}$ Jing Li, ${ }^{1}$ Jonas Haggstrom, ${ }^{2}$ Chee Ng, ${ }^{1}$ Saloumeh Kadkhodayan-Fischer, ${ }^{1}$ Melissa Cheu, \\ Yamo Deniz, ${ }^{3}$ Henry Lowman, ${ }^{4}$ Paul Fielder, ${ }^{1}$ Jennifer Visich, ${ }^{1}$ Amita Joshi, ${ }^{1}$ and Nelson "Shasha" Jumbe ${ }^{1}$
}

Received 20 February 2008; accepted 28 May 2008; published online 7 August 2008

\begin{abstract}
HAE1, a high-affinity anti-IgE monoclonal antibody, is discussed here as a case study in the use of quantitative pharmacology in the development of a second-generation molecule. In vitro, preclinical, and clinical data from the first-generation molecule, omalizumab, were heavily leveraged in the HAE1 program. A preliminary mechanism-based pharmacokinetic/pharmacodynamic (PK/PD) model for HAE1 was developed using an existing model for omalizumab, together with in vitro binding data for HAE1 and omalizumab. When phase I data were available, the model was refined by simultaneously modeling PK/PD data from omalizumab studies with the available HAE1 phase I data. The HAE1 clinical program was based on knowledge of the quantitative relationship between a pharmacodynamic biomarker, suppression of free $\mathrm{IgE}$, and clinical response (e.g., lower exacerbation rates) obtained in pivotal studies with omalizumab. A clinical trial simulation platform was developed to predict free $\operatorname{IgE}$ levels and clinical responses following attainment of a target free IgE level $(\leq 10 \mathrm{IU} / \mathrm{ml})$. The simulation platform enabled selection of four doses for the phase II dose-ranging trial by two independent methods: dose-response non-linear fitting and linear mixed modeling. Agreement between the two methods provided confidence in the doses selected. Modeling and simulation played a large role in supporting acceleration of the HAE1 program by enabling data-driven decision-making, often based on confirmation of projections and/or learning from incoming new data.
\end{abstract}

KEYWORD: anti-IgE, monoclonal antibody, quantitative pharmacology.

\section{INTRODUCTION}

Quantitative pharmacology is a multi-disciplinary approach that integrates data about the biological system, drug characteristics, and disease to translate scientific discoveries into successful therapeutics (1). Integrating knowledge about the biology of the target with data from preclinical studies and the literature may help predict the behavior of a novel therapeutic in humans. Quantitative pharmacology may also be used to develop improved second-generation molecules and to design drug candidates to fit the desired target product profile prior to development.

Modeling and simulation offer powerful tools to perform quantitative pharmacology. The operating paradigm of model development is a continuous cycle of learning, confirming,

\footnotetext{
$\overline{{ }^{1} \text { Development Sciences, Genentech Inc., } 1 \text { DNA Way, South San }}$ Francisco, California 94080-4990, USA.

${ }^{2}$ Design, Analysis, and Technology Administration, Genentech, Inc., 1 DNA Way, South San Francisco, California 94080-4990, USA.

${ }^{3}$ Immunology, Tissue Growth and Repair Clinical Sciences, Genentech, Inc., 1 DNA Way, South San Francisco, California 94080-4990, USA.

${ }^{4}$ Antibody Engineering, Genentech, Inc., 1 DNA Way, South San Francisco, California 94080-4990, USA.

${ }^{5}$ To whom correspondence should be addressed. (e-mail: wputnam @gene.com)
}

and updating throughout the development of a drug candidate. In the learning mode, studies explore the relationships between patient characteristics, dose regimen, efficacy and toxicity; subsequent studies confirm what has been learned in a representative patient population (2).

Since the advent of simulation software systems in the mid-1990s, pharmaceutical companies have been expanding their use of clinical trial simulations (3) to better design clinical trials. Clinical responses for different trial designs may be predicted by resampling subjects from simulated clinical databases using bootstrapping. Quantitative model-based decision-making can help optimize drug development by increasing the probability of technical success, accelerating timelines, and reducing costs $(4,5)$.

The development of HAE1, a high-affinity anti-IgE monoclonal antibody, is a case study in the use of quantitative pharmacology in the development of a second-generation molecule. To inform decision-making, data were integrated from a variety of sources, including characterization studies with HAE1 and an extensive database from the first generation molecule, omalizumab (Xolair ${ }^{\circledR}$ ). The binding characteristics of HAE1 and omalizumab, together with omalizumab clinical data, were used to develop a mechanism-based pharmacokinetic/pharmacodynamic (PK/PD) model, which was used to simulate clinical PK/PD profiles 
to optimize phase I and II trial designs (i.e., dose and regimen selections, number of patients, and endpoint strategy). The trial designs were based on knowledge of the quantitative relationship between a pharmacodynamic biomarker, suppression of free $\mathrm{IgE}$, and clinical response (e.g., lower exacerbation rates) obtained in pivotal studies with omalizumab. A modeling and simulation strategy based on a learnconfirm-update cycle supported data-driven decision-making throughout the HAE1 development program.

\section{HAE1 BACKGROUND}

\section{Mechanism of Action}

After exposure to an allergen, atopic patients produce $\mathrm{IgE}$ antibodies, which bind to FceRI receptors on the surface of mast cells and basophils. An allergic response occurs when allergens crosslink the IgE molecules, degranulating the effector cells and immediately releasing proinflammatory mediators, such as histamine (6). The first recombinant antiIgE therapy, omalizumab (Xolair $\left.{ }^{\circledR}\right)$, was approved by FDA for the treatment of moderate-to-severe asthma in 2003. HAE1 is a second-generation fully humanized monoclonal antibody that binds to the same epitope on $\operatorname{IgE}$ as omalizumab but has a much higher binding affinity. Both HAE1 and omalizumab inhibit the allergic cascade by binding human $\mathrm{IgE}$ and blocking the binding of $\operatorname{IgE}$ to FceRI receptors.

\section{HAE1 Characteristics}

Like omalizumab, approximately $94 \%$ of the HAE1 sequence is derived from human IgG1 and approximately $6 \%$ is derived from a murine anti-IgE monoclonal antibody, mainly in the complementarity-determining regions (CDR). HAE1 has the same IgG1 framework as omalizumab; however, it differs from omalizumab by nine amino acids in the CDR.

In vitro studies with the Fab fragments of HAE1 and omalizumab demonstrated that these nine amino acid changes increased the binding affinity of HAE1 to $\operatorname{IgE}$ by approximately 23-fold over that of omalizumab (Table I). The apparent dissociation constant $\left(K_{\mathrm{d}}\right)$ of HAE1 was calculated as the ratio of the dissociation rate of HAE1 from the HAE1: IgE complexes $\left(k_{\text {off }}\right)$ over its association rate $\left(k_{\text {on }}\right)$. Both omalizumab and HAE1 had similar association rates, but HAE1 dissociated from the complexes at a much slower rate.

The higher-affinity HAE1 antibody offered the potential to expand the patient population for anti-IgE therapy to include subjects with higher baseline $\operatorname{IgE}$ levels and to reduce dose levels or increase dosing intervals relative to current Xolair ${ }^{\circledR}$ therapy. The projected impact on dosing was factored into cost analyses, which supported the economic viability of the program.

\section{PRECLINICAL STUDIES SUPPORTING PROOF OF ACTIVITY}

The functional effects of increasing the binding affinity of HAE1 to IgE were characterized in in vitro pharmacology studies. In competitive binding studies, HAE1 was 5.1- to 21fold and 4.3- to 25-fold more potent than omalizumab in inhibiting human and cynomolgus monkey $\operatorname{IgE}$ binding to Fc $\varepsilon$ RI, respectively (Genentech data on file). In studies with rat basophil cells that had been engineered to express the human FceR1 $\alpha$ subunit (RBL-48 cells), the increased binding affinity of HAE1 improved its ability to inhibit ragweedinduced histamine release approximately 13 -fold over omalizumab (Fig. 1).

Because neither HAE1 nor omalizumab binds to rodent IgE, preclinical efficacy data could not be obtained using in vivo rodent models of asthma. However, after administration of a single dose of HAE1 to cynomolgus monkeys, free $\operatorname{IgE}$ levels decreased in a dose-dependent manner, demonstrating the proof of concept of the molecule (D. Mortensen, et al. The Pharmacokinetics and Pharmacodynamics of an anti-IgE Monoclonal Antibody following Single Subcutaneous Dosing in Cynomolgus Monkey. AAPS Annual Meeting. 2007. Abstract \#T3007). Furthermore, the extensive and relevant safety and efficacy data for omalizumab were leveraged in the development of HAE1.

\section{FREE IGE AS A PHARMACODYNAMIC BIOMARKER}

Clinical studies with omalizumab supported the use of free $\operatorname{IgE}$ as a pharmacodynamic biomarker for the HAE1 program (7). After administration of omalizumab to humans, a reversible increase in total $\mathrm{IgE}$ was observed, with a corresponding reversible decrease in serum free $\mathrm{IgE}$ levels. Cynomolgus monkey studies with omalizumab suggested that the increase in total $\operatorname{IgE}$ was due to a reduction in $\operatorname{IgE}$ clearance after forming omalizumab: $\operatorname{IgE}$ complexes (8). In humans, formation of complexes suppressed circulating free IgE concentrations to levels associated with lower exacerbation rates in asthma patients (7). Omalizumab lowered free $\operatorname{IgE}$ in a dose- and baseline $\operatorname{IgE}$-dependent manner $(9,10)$. Serum free $\operatorname{IgE}$ levels were reduced within one hour postdose, with a mean decrease greater than $96 \%$ using recommended doses (Xolair ${ }^{\circledR}$ Package Insert). The reduction in free $\mathrm{IgE}$ levels was maintained with continued administration.

The HAE1 clinical development plan was based on knowledge of the quantitative relationship between the level of free $\mathrm{IgE}$ suppression and clinical response obtained during

Table I. Kinetic Binding Parameters (Mean \pm SD) of HAE1-Fab and Omalizumab-Fab Fragments to $\operatorname{IgE}$

\begin{tabular}{lccc}
\hline \multicolumn{1}{c|}{ Parameter $^{a}$} & Omalizumab-Fab Value & HAE1-Fab Value & Relative Value $^{b}$ \\
\hline$k_{\text {off }}\left(10^{-4} \mathrm{~s}^{-1}\right)$ & $22.0 \pm 1.1$ & $1.0 \pm 0.18$ & 22 \\
$k_{\text {on }}\left(10^{5} \mathrm{M}^{-1} \mathrm{~s}^{-1}\right)$ & $1.4 \pm 0.10$ & $1.5 \pm 0.10$ & 0.93 \\
$K_{\mathrm{d}}=k_{\text {off }} / k_{\text {on }}(\mathrm{nM})$ & $15.5 \pm 1.3$ & $0.66 \pm 0.13$ & 23 \\
\hline
\end{tabular}

${ }^{a} K_{\mathrm{d}}=$ dissociation constant; $k_{\text {on }}=$ rate of association; $k_{\text {off }}=$ rate of dissociation

${ }^{b}$ Relative value $=($ omalizumab-Fab value $) /($ HAE1-Fab value $)$ 


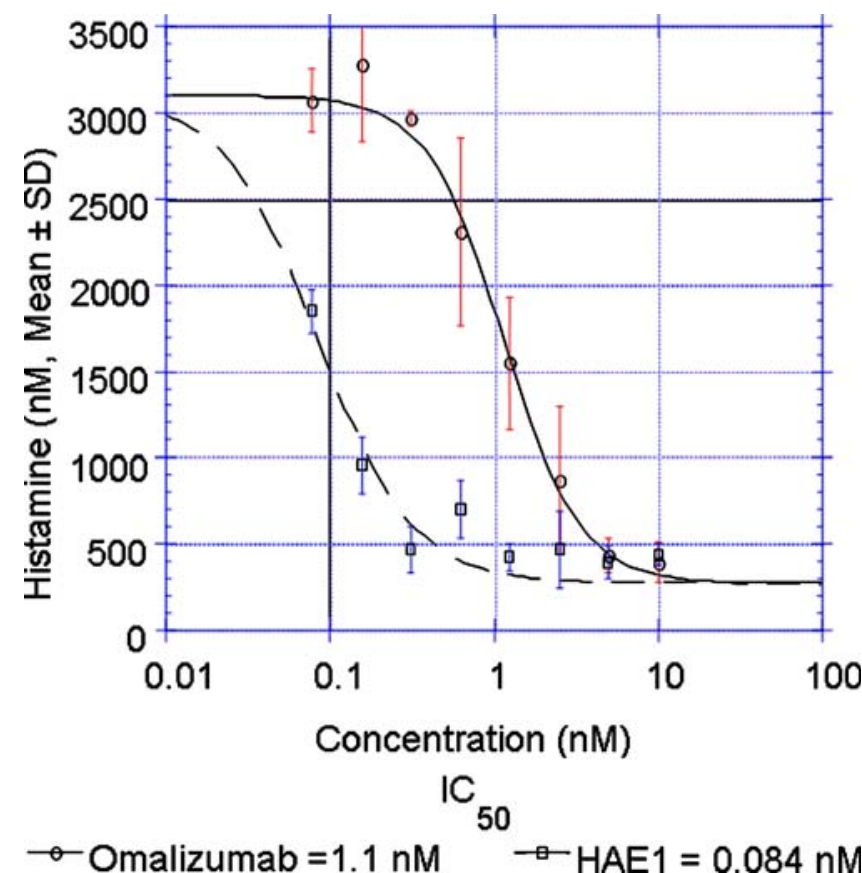

Fig. 1. Inhibition of histamine release from ragweed-specific IgEloaded RBL-48 cells by HAE1 and omalizumab

the development of omalizumab. The omalizumab program established that reducing mean free IgE levels to approximately $10 \mathrm{IU} / \mathrm{ml}$ in asthma patients significantly lowered exacerbation rates (7). Furthermore, a retrospective analysis of two asthma studies showed no additional clinical benefit of suppressing free $\operatorname{IgE}$ below the average target level (7). Based on these findings, the objective of the HAE1 clinical plan was to select a convenient dosing regimen that consistently achieved this target level of free $\operatorname{IgE}$ suppression. Although HAE1 binds IgE at the same locus as omalizumab, due to its higher affinity, the second-generation molecule was expected to require a lower molar ratio of drug to $\operatorname{IgE}$ to achieve the target free IgE level.

\section{MODELING AND SIMULATION APPROACHES FOR PHASE I AND II DESIGNS}

\section{Structure of Mechanism-Based PK/PD Model}

The overall PK/PD modeling strategy for the HAE1 clinical development program follows. To determine doses for the phase I study, a preliminary HAE1 PK/PD model was developed using an existing PK/PD model for omalizumab, together with in vitro binding data for HAE1. When phase I data were available, the preliminary model was refined by simultaneously modeling $\mathrm{PK} / \mathrm{PD}$ data from omalizumab clinical studies with the available HAE1 phase I data to develop a refined HAE1 PK/PD model. The refined model was then used to perform an extensive clinical trial simulation exercise to evaluate projected dose-response profiles for various clinical responses (e.g., asthma symptoms). The clinical trial simulations enabled selection of doses for the HAE1 phase II study. As phase II data became available, the model could be further refined to select phase III doses.

The first step in the modeling strategy was the development of a mechanism-based omalizumab PK/PD model using data from three phase I studies. The dataset included a total of 134 patients and 9,349 samples from single-dose and multiple-dose studies. The structure of the model (Fig. 2) was similar to models developed by Hayashi et al. (11) and Y-N. Sun (Advanced Methods of PK/PD Systems Analysis, Biomedical Simulations Resource Workshop, Marina del Rey, CA, June 22-23, 2001). In Fig. 2, CL $\mathrm{L}_{\text {Drug }}$ is the clearance for a free anti-IgE monoclonal antibody, while $\mathrm{CL}_{\mathrm{E}}$ and $\mathrm{CL}_{\mathrm{X}}$ are the clearances for the free IgE and IgE-drug complex, respectively. FcRn refers to the Fc $\gamma$ neonate receptor, Fce is the $\mathrm{Fc} \varepsilon$ receptor pathway, and $\mathrm{Fc} \gamma$ is the $\mathrm{Fc} \gamma$ receptor pathway. $K_{\mathrm{syn}}$ is the zero-order synthesis rate constant for IgE.

Although several receptor binding models have been previously described (12), the choice of the basic model structure was based on known receptor-occupancy physiology. This physiologic binding model is based on the law of mass-action and is applicable for calculating the inhibition of free target in order to answer questions as to what dose, regimen, and affinity are required to bind the target between dosing intervals.

The omalizumab PK/PD model described the relationship between the pharmacokinetics of omalizumab, the reversible binding of omalizumab with free $\operatorname{IgE}$ and the disposition of free $\operatorname{IgE}$ and omalizumab: $\operatorname{IgE}$ complexes. Although free IgE clears rapidly from the circulation by endocytosis and binding to Fce receptors (6), omalizumab is protected from endocytosis by recycling through the IgG salvage receptor, FcRn (13). The clearance of omalizumab is also controlled by non-specific IgG clearance and reversible binding to free IgE. Omalizumab forms relatively small, biologically inert complexes with $\operatorname{IgE}(7)$, which clear through Fc $\gamma$ receptors of the reticuloendothelial system (8).

Given the similarity in structure and behavior between HAE1 and omalizumab in in vitro and in vivo nonclinical studies, it was expected that the disposition of HAE1 in humans would be similar to that of omalizumab. Hence, the mechanism-based PK/PD model developed for omalizumab could be adopted for HAE1. A preliminary HAE1 PK/PD model was generated by replacing the in vivo dissociation constant $\left(\mathrm{K}_{\mathrm{d}}\right)$ for omalizumab:IgE binding with the in vivo $K_{\mathrm{d}}$

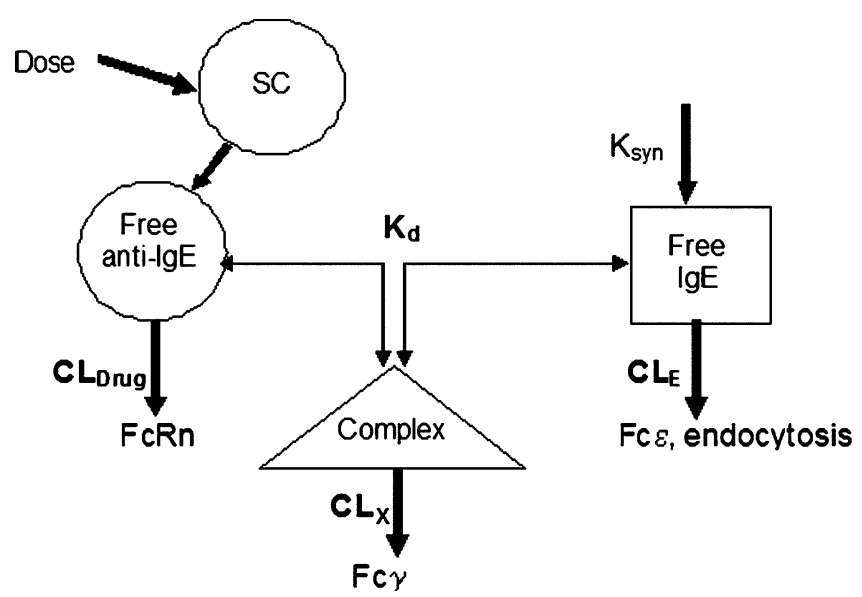

Fig. 2. Structure of mechanism-based $\mathrm{PK} / \mathrm{PD}$ model for an anti-IgE monoclonal antibody 
for HAE1: IgE binding in the omalizumab model. This analysis required three key assumptions: (1) the observed omalizumab and HAE1 in vitro and in vivo $K_{\mathrm{d}}$ ratios were assumed to be directly proportional; (2) the PK and PD parameters were similar for omalizumab and HAE1, except for the $K_{\mathrm{d}}$, and (3) PK/PD covariates (specifically baseline IgE and body weight) were similar for omalizumab and HAE1.

\section{HAE1 Phase I Study Design}

The preliminary HAE1 model was used to simulate HAE1 PK/PD profiles in the phase I study, which investigated a single dose, dose-escalation of HAE1 in adults with allergic rhinitis with or without atopic dermatitis. PK/PD simulations were conducted to select the phase I doses by determining the range of subcutaneous doses given every 4 weeks to achieve a reduction of average serum free $\operatorname{IgE}$ to $\leq 10 \mathrm{IU} / \mathrm{ml}$ in the target patient population. Because the dosing of omalizumab is based on the patient's baseline $\mathrm{IgE}$ level and body weight, simulations explored a broad range of body weights $(40-150 \mathrm{~kg})$ and baseline IgE levels (20-1,500 IU/ml). Although the target upper limit for the simulation exercise was set at $1,500 \mathrm{IU} / \mathrm{ml}$ for pretreatment $\mathrm{IgE}$ levels, patients with even higher levels, up to $3,000 \mathrm{IU} / \mathrm{ml}$, were allowed to participate in the phase I study in order to get a robust dataset for refinement of the preliminary HAE1 PK/PD model.

In the dose-escalation phase of the study, patients were randomized into three dosing cohorts: 30/90, 180, and $360 \mathrm{mg}$.
In the first cohort, a minimum pharmacologic dose of $30 \mathrm{mg}$ HAE1 was initially given to a subcohort of patients to ensure that injections were well tolerated before escalating to the $90 \mathrm{mg}$ dose. PK/PD data from the subjects were analyzed as samples were received during the study to assess the performance of the drug and allow adjustments to the study design. When PK/PD data from the lower cohorts indicated that free IgE suppression was greater than expected based on simulated HAE1 profiles and predictions for higher-affinity anti-IgE antibodies (5), the study protocol was amended and a lower $7.5 \mathrm{mg}$ dose was added in order to fully characterize the HAE1 dose-response profile.

Figure 3 shows the PK/PD profiles for five phase I subjects who received HAE1 doses of 7.5, 30, 90, 180 or $360 \mathrm{mg}$. HAE1 concentrations showed the expected profile for subcutaneous absorption and elimination. Even at the $7.5 \mathrm{mg}$ dose, free IgE levels were suppressed below the target free $\mathrm{IgE}$ level of $\leq 10 \mathrm{IU} / \mathrm{ml}$ in this subject. As the dose increased, the duration of suppression also tended to increase. As free $\mathrm{IgE}$ levels decreased, total $\mathrm{IgE}$ levels increased, consistent with the hypothesis that HAE1:IgE complexes cleared at a slower rate than free $\mathrm{IgE}$ alone. As expected, free and total IgE levels returned to baseline levels.

\section{HAE1 Phase II Study Design}

To enable multidose HAE1 PK/PD predictions and to provide study design and decision-making support for the phase II study in asthma patients, the preliminary HAE1 PK/ PD model was refined using the phase I data. The refined
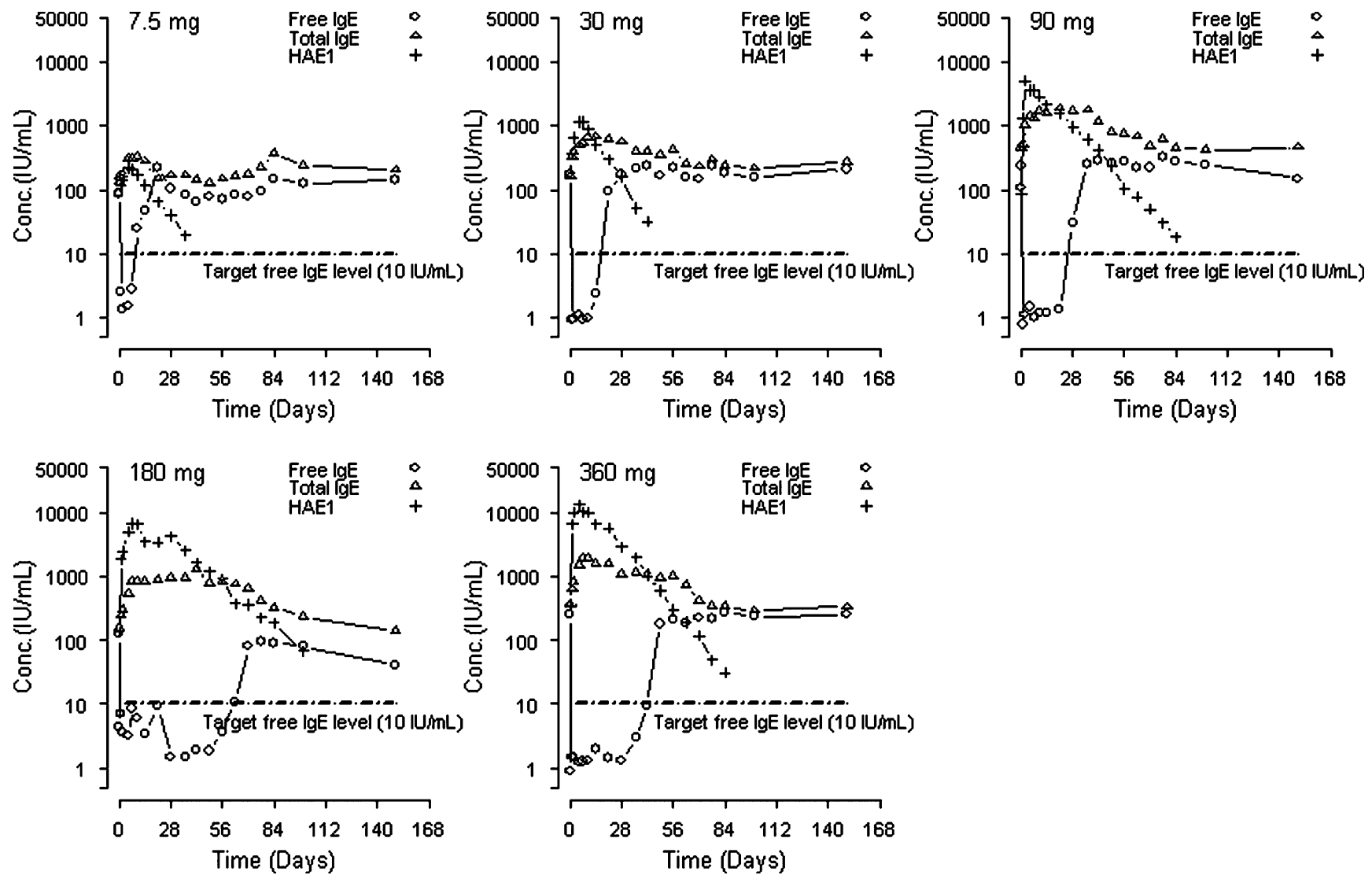

Fig. 3. HAE1, total IgE, and free IgE concentration-time profiles for five HAE1 subjects who received a single subcutaneous dose of 7.5, 30, 90,180 or $360 \mathrm{mg}$ 


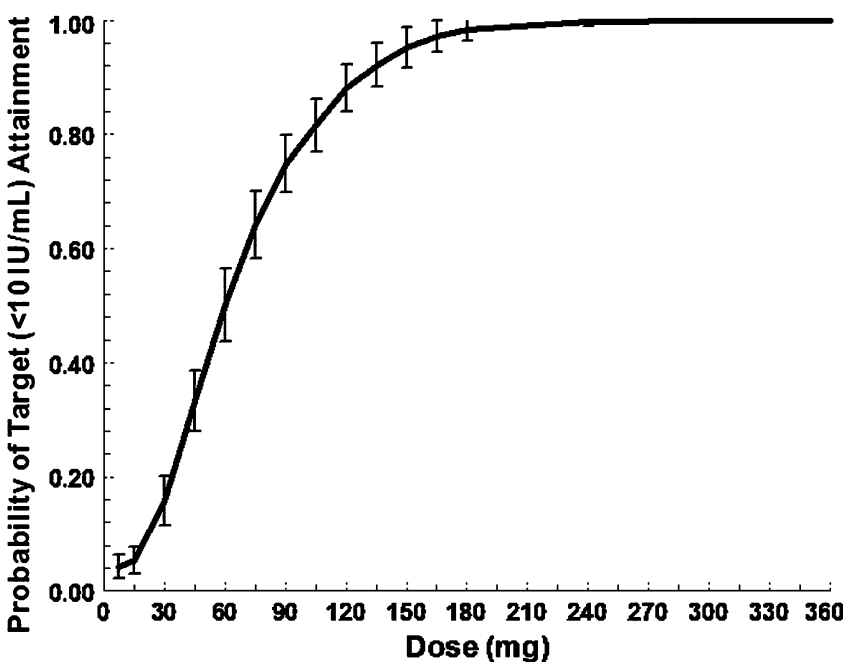

Fig. 4. Simulated probability of HAE1-treated subjects achieving target free IgE level of $\leq 10 \mathrm{IU} / \mathrm{ml}$ as a function of dose

HAE1 PK/PD model was developed by simultaneously modeling PK/PD data from omalizumab clinical studies and available data from the HAE1 phase I study. The underlying assumption for this approach was that given the observed similarity in the structure, behavior, and mechanism of action for omalizumab and HAE1, kinetic information on free $\operatorname{IgE}$ could be reliably shared between the two molecules, while elimination of free drug by FcRn and IgE complexes by Fc $\gamma$ receptors would be molecule-specific. Additionally, parameterization of the preliminary HAE1 PK/PD model was modified in the refined HAE1 PK/PD model to explicitly include body weight and baseline $\operatorname{IgE}$ variables for both molecules to permit subsequent evaluation of the impact of these variables on outcomes.

A population $\mathrm{PK} / \mathrm{PD}$ analysis was performed using NONMEM (Version V, level 1.1) based on 8,565 and 914 serum concentration samples from 105 and 25 patients who received omalizumab or HAE1, respectively. A non-parametric bootstrap procedure (M. R. Gastonguay and A. El-Tahtawy. Effect of NONMEM minimization status and number of replicates on bootstrap parameter distributions for population pharmacokinetic models: A case study. Clin Pharmacol Ther. 77: P2-P2. 2005) was used to estimate the precision of model parameters, and the model performance was assessed using a visual predictive check.

Two independent methods were used to select doses for the phase II dose-ranging trial. Both methods used a clinical trial simulation platform, which predicted free IgE levels and clinical responses following attainment of target free $\operatorname{IgE}$ levels. Dose-response profiles were subsequently evaluated using nonlinear fitting methods to approximate effective doses (e.g., $\mathrm{ED}_{5}, \mathrm{ED}_{25}, \mathrm{ED}_{50}$, and $\mathrm{ED}_{90}$ ) and by selecting equally spaced doses following linear mixed modeling to evaluate a trend in the predicted clinical response. Although various aspects of clinical response may be assessed in asthma patients, such as exacerbation rates and lung function, these approaches examined the total symptom score, which accounts for both daytime and nocturnal asthma symptoms. Agreement between the two independent methods provided confidence in the doses selected.
The clinical trial simulation platform encompassed the following elements:

- Model parameters and associated between-subject variance estimates from the refined HAE1 PK/PD model were fixed to their final values.

- Target patient population demographics (body weight and baseline $\mathrm{IgE}$ ) were defined based on baseline values observed in ongoing omalizumab studies targeting the same population.

The following study design elements were incorporated in the simulation:

- HAE1 treatment was once every four weeks by subcutaneous administration as a fixed dose or normalized by body weight and baseline IgE.

- A subject withdrawal rate $(\sim 0.7 \% /$ week $)$ was randomly implemented to coincide with the observed drop-out rates in the omalizumab clinical trials.

- Other protocol elements, such as clinic visits, sample collection times, free IgE levels, and clinical response were also incorporated.

A 5,000-subject clinical trial database was created by clinical trial simulation. Fifty bootstrap iterations with resampling of 100 subjects were used to perform three simulation exercises to determine (1) the probability of attaining the target free $\mathrm{IgE}$ level at a given dose at any given time during the dosing interval, and following attainment of the target free IgE level, (2) the probability of achieving the target clinical response (e.g., change in total symptom score), and (3) the projected proportion of simulated subjects with reduced total symptom scores.

The first PK/PD simulation exercise involved determining the probability of treated subjects achieving the target free $\mathrm{IgE}$ level of $\leq 10 \mathrm{IU} / \mathrm{ml}$. This approach showed that increasing the HAE1 dose increased the probability of subjects achieving the target free IgE level (Fig. 4). Further

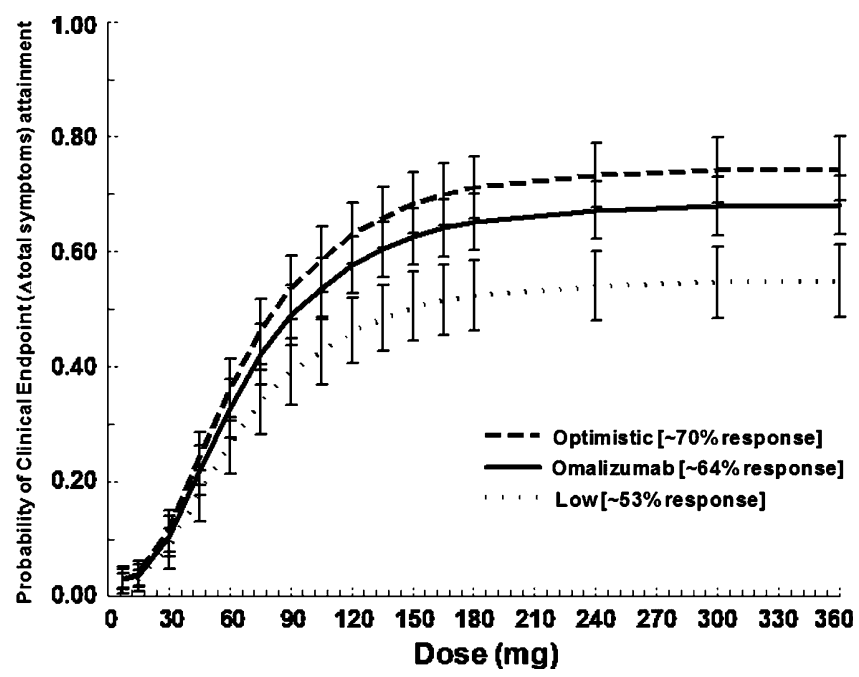

Fig. 5. Simulated probability of attaining clinical response ( $\Delta$ total symptom score) as a function of dose for three cases: an optimistic response rate $(70 \%)$, a response rate similar to that expected for omalizumab $(64 \%)$, and a low response rate $(50 \%)$ 
increases in HAE1 dose beyond $180 \mathrm{mg}$ were not expected to improve the response.

The second simulation exercise predicted the probability of attaining a clinical response (e.g., change in total symptom score) as a function of dose. This exercise used a global approach whereby fixed proportions of subjects attaining the target free $\mathrm{IgE}$ level of $\leq 10 \mathrm{IU} / \mathrm{ml}$ as a function of dose were presumed to have clinical benefit. Data from the literature (14) based on predictors of response analyses for omalizumab were used to transition from free IgE suppression (a pharmacodynamic biomarker for treatment response) to the clinical efficacy domain. Figure 5 shows the dose versus projected clinical response curves for three cases: a response rate similar to that expected for omalizumab (64\%), an optimistic rate $(70 \%)$, and a low response rate $(50 \%)$. The high and low response rates were clinical response rates from retrospective analyses of omalizumab data (14). The doseresponse curves for probability of target free $\mathrm{IgE}$ suppression (Fig. 4) or probability of clinical response (as a change in total symptom score, Fig. 5) were similar and suggested selection of identical phase II doses.

An alternative approach for evaluating treatment effect is by the odds ratio. In order to examine what the doseresponse would look like when constrained based on the observed omalizumab/placebo odds ratio and the extent of reduction in total symptom scores, a third simulation exercise was undertaken. This exercise was informed by omalizumabvs.-placebo odds ratio analyses (14) and observed treatment effects $(-1.62 \pm 1.6$ and $-1.15 \pm 1.6$ for omalizumab and placebo, respectively). This exercise was most useful in gaining further insight into the lower portion of the dose-response relationship and helped identify the lowest dose selected for the phase II study.

An alternative method used for parallel independent selection of the phase II doses was linear mixed modeling (15). This approach was used to evaluate a trend in response across the simulated doses using a linear contrast in leastsquares means, as well as a comparison between the different doses using differences in least-squares means. Doses selected by the two independent methods, dose-response nonlinear fitting and linear mixed modeling, were placebo, 7.5-15, 3045, 90, and $180 \mathrm{mg}$.

Based on these analyses, four doses were selected for the phase II study: a "minimal effect" dose of $15 \mathrm{mg}$, two intermediate doses, 45 and $90 \mathrm{mg}$, and a high dose of $180 \mathrm{mg}$ near the top of the dose-response curve.

\section{CONCLUSIONS}

A quantitative pharmacology platform was successfully incorporated into the development of HAE1, from the in vitro characterization and preclinical studies through the clinical program. This model-based approach was used as a tool to communicate with the development team and to clarify risks and assumptions during the design of the phase I and II studies. When phase I data became available, a preliminary $\mathrm{PK} / \mathrm{PD}$ model was refined and used to make predictions to enable dose selection for the subsequent study. Simulation approaches were used to aid interpretation of incoming clinical results, to give insights into complex PK/PD interactions, and to estimate the relationship between HAE1 dose and a pharmacodynamic biomarker, suppression of free IgE levels. Incorporating a modeling approach early in the development of HAE1 allowed the project team to proceed with confidence from phase I to a well-designed phase II dose-finding study using the minimum number of subjects. Although the HAE1 program was discontinued due to two hypersensitivity reactions in the phase II study, modeling and simulation played a large role in supporting acceleration of the program by enabling data-driven decision-making, often based on confirmation of projections and/or learning from incoming data.

\section{REFERENCES}

1. E. I. Ette, and P. J. Williams. Pharmacometrics: The Science of Quantitative Pharmacology, Wiley, New Jersey, 2007.

2. L. B. Sheiner. Learning versus confirming in clinical drug development. Clin. Pharmacol. Ther. 61:275-291 (1997).

3. P. L. Bonate. Pharmacokinetic-Pharmacodynamic Modeling and Simulation, Springer Science, New York, 2006.

4. R. L. Lalonde, K. G. Kowalski, M. M. Hutmacher, W. Ewy, D. J. Nichols, P. A. Milligan, B. W. Corrigan, P. A. Lockwood, S. A Marshall, L. J. Benincosa, T. G. Tensfeldt, K. Parivar, M. Amantea, P. Glue, H. Koide, and R. Miller. Model-based drug development. Clin. Pharmacol. Ther. 82:21-32 (2007).

5. B. M. Agoram, S. W. Martin, and P. H. van der Graaf. The role of mechanism-based pharmacokinetic-pharmacodynamic (PKPD) modelling in translational research of biologics. Drug Discov. Today. 12:1018-1024 (2007).

6. T. Platts-Mills. Hypersensitivity-Type I. In I. Roitt, J. Brostoff, and D. Male (eds.), Immunology, Mosby, London, 2001, pp. 323327.

7. G. Hochhaus, L. Brookman, H. Fox, C. Johnson, J. Matthews, S. Ren, and Y. Deniz. Pharmacodynamics of omalizumab: implications for optimized dosing strategies and clinical efficacy in the treatment of allergic asthma. Curr. Med. Res. Opin. 19:491-498 (2003).

8. J. A. Fox, T. E. Hotaling, C. Struble, J. Ruppel, D. J. Bates, and M. B. Schoenhoff. Tissue distribution and complex formation with IgE of an anti-IgE antibody after intravenous administration in cynomolgus monkeys. J. Pharmacol. Exp. Ther. 279:10001008 (1996).

9. L. P. Boulet, K. R. Chapman, J. Cote, S. Kalra, R. Bhagat, V. A. Swystun, M. Laviolette, L. D. Cleland, F. Deschesnes, J. Q. Su, A. DeVault, R. B. Fick Jr., and D. W. Cockcroft. Inhibitory effects of an anti-IgE antibody E25 on allergen-induced early asthmatic response. Am. J. Respir. Cri. Care Med. 155:1835-40 (1997).

10. T. B. Casale, I. L. Bernstein, W. W. Busse, C. F. LaForce, D. G. Tinkelman, R. R. Stoltz, R. J. Dockhorn, J. Reimann, J. Q. Su, R. B. Fick Jr., and D. C. Adelman. Use of an anti-IgE humanized monoclonal antibody in ragweed-induced allergic rhinitis. $J$. Allergy Clin. Immunol. 100:110-121 (1997).

11. N. Hayashi, Y. Tsukamoto, W. M. Sallas, and P. J. Lowe. A mechanism-based binding model for the population pharmacokinetics and pharmacodynamics of omalizumab. Br. J. Clin. Pharmacol. 63:548-561 (2007).

12. W. D. M. Paton. A theory of drug action based on the rate of drug-receptor combination. Proc. Roy Soc. Lond. B Biol. Sci. 154:21-69 (1961).

13. V. Ghetie, J. G. Hubbard, J.-K. Kim, M.-F. Tsen, Y. Lee, and E. S. Ward. Abnormally short half-lives of IgG in $\beta_{2}$-microglobulindeficient mice. Eur. J. Immunol. 26:690-696 (1996).

14. J. Bousquet, S. Wenzel, S. Holgate, W. Lumry, P. Freeman, and H. Fox. Predicting response to omalizumab, an anti-IgE antibody, in patients with allergic asthma. Chest. 125:1378-1386 (2004).

15. J. Chen, D. Zhang, and M. Davidian. A Monte Carlo EM algorithm for generalized linear mixed models with flexible random effects distribution. Biostatistics. 3:347-360 (2002). 